\title{
Traffic Characteristics in Adaptive Prioritized-Handoff Control Method Considering Reattempt Calls
}

\author{
Noriteru Shinagawa ${ }^{1}$, Takehiko Kobayashi, ${ }^{1}$, \\ Keisuke Nakano ${ }^{2}$, and Masakazu Sengoku ${ }^{2}$ \\ ${ }^{1}$ YRP Mobile Telecommunications Key Technology Research Laboratories Co., Ltd. \\ YRP Center, Ichibankan, 6F 3-4 Hikari-no-oka, Yokosuka, 239-0847 Japan \\ \{shina,koba\} @yrp-ktrl.co.jp \\ ${ }^{2}$ Faculty of Engineering, Niigata University \\ 2-8050, Ikarashi, Niigata, 950-2181 Japan \\ \{nakano, sengoku\}@ie.niigata-u.ac.jp
}

\begin{abstract}
In a cellular mobile communications system, the call in progress will be forcibly terminated, if a circuit to the destination base station cannot be secured when a handoff is attempted. Studies have therefore been performed on methods of decreasing the percentage of forcibly terminated calls by giving priority to handoff calls when the circuits are allocated. In these studies, associated traffic characteristics have been examined based on the assumption that blocked calls simply disappear. This paper proposes an adaptive handoff priority control method that varies the number of reserved circuits for handoff based on the measured of handoff blocking rate, and evaluates traffic characteristics of the proposed method and conventional one for the cases that consider and ignore reattempt calls. It was found that the proposed method could mitigate the effects of change in average speed of mobile stations and in reattempt-call parameter such as maximum retry number.
\end{abstract}

\section{Introduction}

When a mobile station (MS) with a call in progress moves across cell boundary in a cellular mobile communications system, the system performs circuit switching from the base station (BS) within the current cell to the BS within the destination cell to enable uninterrupted communications. This process is called "handoff." At this time, however, if a circuit to the destination BS cannot be secured, the call will be forcibly terminated. To therefore continue communicating, the connection process must be repeated, which, of course, degrades service quality. Against this background, studies have been performed on methods of decreasing forced terminations during handoff by giving handoff calls priority. This can be achieved, for example, by securing a fixed number of circuits especially for handoff, or by having the handoff process wait until a circuit to the destination BS becomes available [1-5]. These studies have been examining traffic characteristics under the condition that calls simply disappear when either blocked or forcibly terminated due to lack of available circuits to the destination BS during connection or handoff processing. It can also be considered, 
though, that users will often try to reconnect (reattempt call) when blocked at a new call attempt or forcibly terminated during a call.

Furthermore, when designing and operating an actual cellular system that employs a handoff priority system by securing a portion of circuits for handoff, an appropriate number of circuits for this purpose must be selected and set at each BS according to various criteria. These include the number of circuits installed at one's own BS and at adjacent BSs and the average speed of MSs that are currently connected within the cell. In addition, considering that the ratio of pedestrians and automobiles making up MSs changes over time and that speed of MS movement varies due to traffic congestion, the system must provide a flexible response to fluctuation in handoff traffic. To meet these demands, this paper proposes a handoff priority control method that adjusts the number of handoff circuits based on the measured number of failed handoffs. And we evaluate traffic characteristics of the proposed method and conventional one that reserve a fixed number of handoff circuits for both the case that assumes reattempt calls and the case that does not.

\section{Proposal for an Adaptive Prioritized-Handoff Control Method}

There are basically two types of methods for giving priority to handoff calls, as follows:

(1) Perform wait processing when all BS circuits are occupied at the time of new call attempt or handoff processing, and when a circuit becomes available, give priority in connection to a queued handoff call.

(2) Denoting the number of circuits at each BS as $C$ and the number of handoffdedicated circuits as $C_{h}$, connect a new call requesting connection only if the number of available circuits is greater than $C_{h}$. A handoff call is given priority in connection by simply connecting it if a circuit is available.

In this paper, we examine the second method that reserves circuits for handoff calls, and propose a method that controls the number of handoff circuits in accordance with the handoff traffic load so that fluctuating handoff traffic can be flexibly handled. The control sequence of the proposed method is summarized below.

For each accommodated base station, a switch incorporates the following counters: A circuit counter $(C)$ that stores the number of circuits installed in the base station; A handoff circuit counter $\left(C_{h}\right)$ that stores the number of circuits reserved for handoff circuits; A free-circuit counter $\left(C_{f}\right)$ that stores the number of free circuits; A counter $\left(C_{n h}\right)$ that counts the number of handoff control events from adjacent cells; and $\mathrm{A}$ handoff-block counter $\left(C_{n b}\right)$ that counts the number of times a circuit could not be secured at the time of handoff control.

A switch will also store threshold values $B_{1}$ and $B_{2}$ (where $B_{1}>B_{2}$ ) as control criteria. On receiving a connection-control request, the switch checks the counters of the base station making the request, and if $C_{f}>C_{h}$, it executes connection control and decrements the value of $C_{f}$ by one, while if $C_{f} \leq C_{h}$, it blocks the new call. On the other hand, when receiving a handoff-control request, the switch increments the value of $C_{n h}$ by one and checks the value of $C_{f}$. If $C_{f}>0$ at this time, the switch executes handoff control and decrements the value of $C_{f}$ by one, while if $C_{f}=0$, it performs a forced termination and increments the value of $C_{n b}$ by one. In relation to the above, an interrupt is generated at regular intervals $(T)$ in the switch, and on detecting the 
interrupt, the switch calculates handoff block rate $B \quad\left(B=C_{n b} / C_{n h}\right)$ for each accommodated base station. Then, if the calculated value of $B$ is less than predetermined threshold $B_{1}$ and if $C_{h}>0$, the value of $C_{h}$ is decremented by one. Conversely, if the calculated value of $B$ is greater than predetermined threshold $B_{2}$ and if $C_{h}<C$, the value of $C_{h}$ is incremented by one. Finally, after completing this processing for all base stations, the switch resets $C_{n h}$ and $C_{n b}$ of each base station to zero. In this way, the number of circuits reserved for handoff can be automatically adjusted by taking periodic measurements of the circuit block rate at the time of handoff control.

\section{Simulation Model}

Simulated evaluation was performed using an endless 10-by-10-cell virtual configuration, where each cell is square-shaped with side of length $L$ and every side is connected to the adjacent cell. The number of circuits set up in each cell was uniform at $C$ circuits, and the call arrival interval followed an exponential distribution. When the time arrives for generating a new call, a uniform random number is used to determine the coordinates, speed $v$, and direction $\theta$ of the MS beginning the call so that calls come to be uniformly distributed within each cell. Here, speed and direction of the MS do not change for the duration of the call. Holding time follows an exponential distribution with an average of 120 seconds.

A newly generated call will be connected to the BS if the number of available circuits in the BS is greater than $C_{h}$. Then, if a connection cannot be made, a connection request will be made again after time $t$. It is assumed that the MS continues to move during this time, and that it will make the next connection request in the cell that it finds itself at that point in time. Finally, if a connection cannot be made after $N$ tries, it is assumed that the MS gives up its attempt to make the call. The time $t$ indicating the interval from the failed connection request to the next retry follows an exponential distribution with an average of 10 seconds.

In the event that a connected call moving at speed $v$ and in direction $\theta$ as determined at call-generation time arrives at an adjacent cell, handoff is performed if a circuit is available at the destination BS. If there is no available circuit, the call is forcibly terminated. In the case of forced termination, a connection request will be retried after $t$ seconds in an attempt to continue the call for the portion of holding time remaining. A reconnection attempt is treated the same as a newly generated call. If a reconnection is achieved, the call continues only for the time determined by subtracting the time already used up before disconnection from the holding time set at call generation. This simulation model that takes reattempt calls into account is shown in Fig. 1 and simulation conditions are listed in Table 1. 


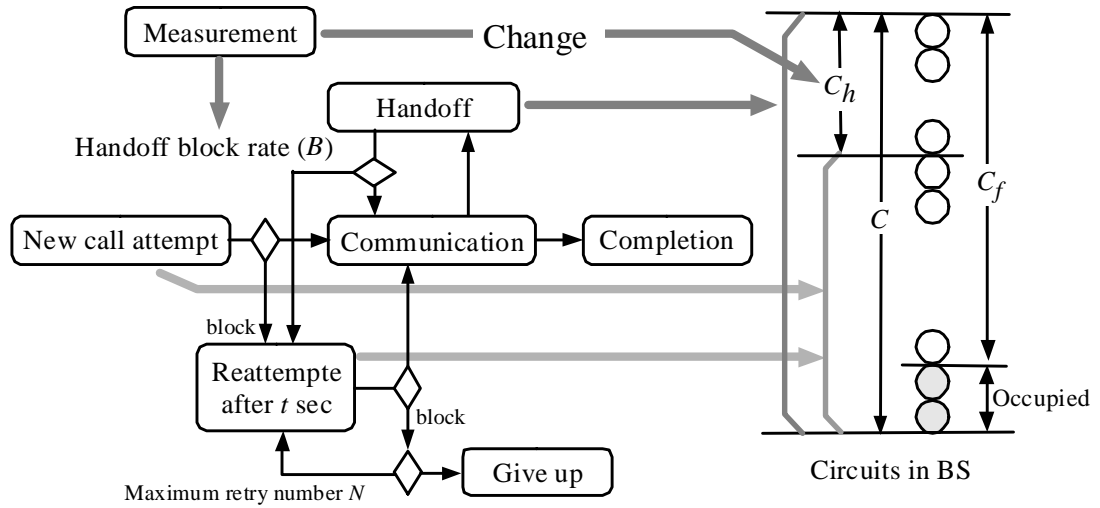

Fig. 1. Simulation model.

Table 1. Simulation conditions

\begin{tabular}{ll}
\hline Cell size & $500 \mathrm{~m}$ \\
Number of circuits in cell & 20 \\
Number of reserved circuits for handoff & $0,2,4$, automatic \\
\hline Speed of mobile station & $0-60 \mathrm{~km} / \mathrm{h}$ \\
Move direction of mobile station & $0-2 \pi \quad$ (uniform distribution) \\
\hline Mean holding time & $120 \mathrm{~s} \mathrm{(exponential} \mathrm{distribution)}$ \\
Mean retry interval & $10 \mathrm{~s} \mathrm{(exponential} \mathrm{distribution)}$ \\
Maximums retry number & $0,5,10,15,20$ \\
\hline Control period & $600 \mathrm{~s}$ \\
$B_{1}$ & 0.02 \\
$B_{2}$ & $0.07,0.15$ \\
\hline
\end{tabular}

\section{Evaluation Measures}

When taking reattempt calls into account, calls that fully complete can be classified into the following two types: (1) calls that complete without being forcibly terminated at handoff after the call began; and (2) calls that complete after reconnecting following a forced termination at the time of handoff. In contrast, when not taking reattempt calls into account, calls that complete would fall only into the first category. Considering, therefore, that forced terminations have a significant effect on service quality, we evaluated call-completed rate $R_{c 1}$ corresponding to calls that experienced no forced termination and call-completed rate $R_{c 2}$ that includes forcibly terminated calls. These two types of call-completed rates are defined as follows.

$$
\begin{aligned}
& R_{c 1}=C_{1} / C_{n} \\
& R_{c 2}=\mathrm{C}_{2} / C_{n}
\end{aligned}
$$

Here, $C_{1}$ is the number of calls that completed without being forcibly terminated, $C_{2}$ is the number of calls that completed both without being forcibly terminated and after 
reconnecting following a forced termination, and $C_{n}$ is the total number of newly generated calls.

We evaluated the "average number of connection successes" to reflect how many times a successful connection is achieved per newly generated call. A higher value for the average number of connection successes means that there are more calls that have reconnected after being forcibly terminated. On the other hand, a value less than unity means that there are many calls that have given up trying to achieve communications without making even one successful connection. The average number of connection successes is denoted as $A_{s}$ and defined as follows:

$$
A_{s}=N_{s} / C_{n}
$$

Here, $N_{s}$ is the number of connection successes and $C_{n}$ is the total number of newly generated calls.

\section{Simulation Results}

The following presents the results of computer simulations for the evaluation measures described in Sect. 4 against the traffic load within the cells, the maximum retry number, and the speed of mobile station communicating in a cell. The symbols used in Figs. 2 to 7 are defined in Table 2.

Table 2. Difinition of symbols used in Figs. 2 to 7

\begin{tabular}{llcc}
\hline & & $\begin{array}{c}\text { Taking no account } \\
\text { of reattempt call }\end{array}$ & $\begin{array}{c}\text { Taking account } \\
\text { of reattempt call }\end{array}$ \\
\hline Conventional method & $C_{h}=0$ & --- & \\
& $C_{h}=2$ & --- & - \\
Proposed method & $C_{h}=4$ & --- & $-\checkmark-$ \\
& $B_{2}=0.07$ & & -- \\
& $B_{2}=0.15$ & & \\
\hline
\end{tabular}

\subsection{Call-Completed Rates}

\section{(a) Characteristics as Function of Traffic Load}

When not taking reattempt calls into account, all calls that complete are calls that do so without being forcibly terminated during a call. In this case, the call-completed rate $\left(R_{c 1}\right)$ gradually drops as traffic load increases. In addition, for the same traffic load, $R_{c 1}$ becomes lower as the number of circuits reserved for handoff becomes larger. This is because more handoff circuits means less circuits that can be used for connecting new calls, that is, the number of calls that are blocked when attempting a connection increases.

When taking reattempt calls into account, the call-completed rate $\left(R_{c 1}\right)$ for only calls that do not experience a forced termination becomes higher as the number of circuits reserved for handoff becomes larger for the same traffic load, which is opposite the characteristics shown when not considering reattempt calls. Here, despite the fact that there are less circuits for connecting new calls, connection can be 
achieved by reconnecting, and the probability of forced termination becomes smaller once a connection is made. In addition, for small traffic load, $R_{c 1}$ shows a higher value than that when not considering reattempt calls, but for larger traffic load, it shows a lower value. This is because a larger traffic load means that circuits must carry more traffic for calls attempting reconnection than that when not considering reattempt calls, which in turn increases the probability of being blocked at handoff. As a characteristic of the proposed system, more handoff circuits are reserved as traffic volume increases in a cell. Next, the call-completed rate $\left(R_{c 2}\right)$ that includes reattempt calls is about unity up to a certain traffic load. In other words, most calls are eventually completed by reconnecting. The $R_{c 2}$, however, begins to drop at a certain traffic load. This drop begins at smaller traffic loads as the number of handoff circuits increases. The proposed method exhibits similar characteristics with the case of reserving four circuits for handoff. For heavy offered traffic, $R_{c 1}$ is small for large values of $C_{h}$ while $R_{c 2}$ is large, i.e., $R_{c 1}$ and $R_{c 2}$ have a tradeoff relationship. For the proposed system, if $B_{2}$ is set to a large value, $R_{c 1}$ decreases while $R_{c 2}$ increases. In this relationship between $R_{c 1}$ and $R_{c 2}$, more importance can be attached to one or the other by adjusting the value of $B_{2}$. Call-completed rates versus traffic load are shown in Fig. 2.

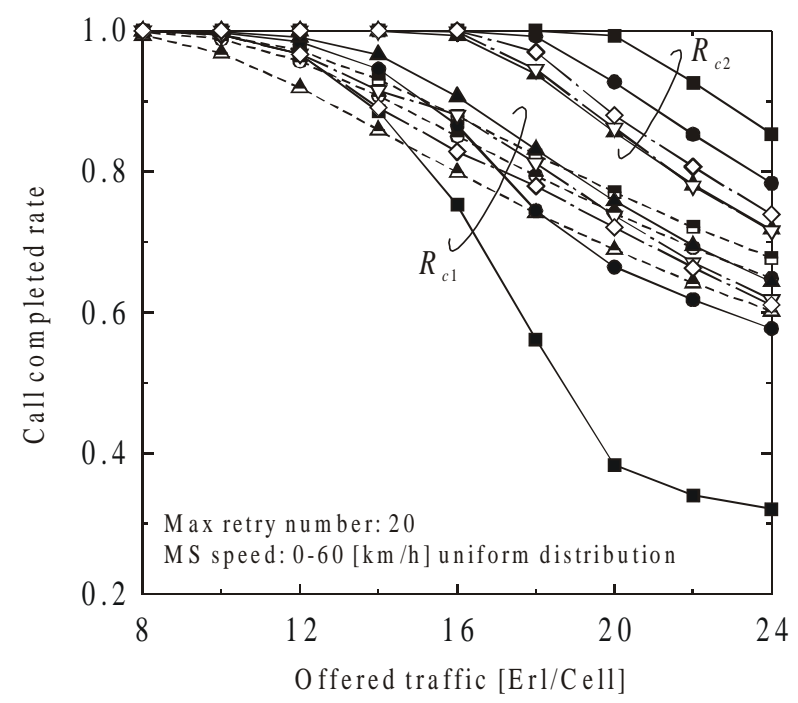

Fig. 2. Call-completed rates versus traffic load.

\section{(b) Characteristics as Function of MS Speed}

When not taking reattempt calls into account, the $R_{c 1}$ drops, if only slightly, as the MS picks up speed. The reason for this is that as speed increases, the frequency of handoffs likewise increases and the probability of being forcibly terminated becomes higher. For the same speed, the $R_{c 1}$ becomes lower as the number of circuits reserved for handoff increases.

When taking reattempt calls into account, the $R_{c 1}$ becomes lower as the number of handoff circuits increases at low speeds. As speed increases, however, $R_{c 1}$ is higher for 
more circuits reserved for handoff. Also, for a fixed number of handoff circuits, $R_{c 1}$ tends to increase with increase in speed up to a certain speed, but then decreases with further increase in speed. This can be attributed to the following. As handoff circuits increase, circuits that can be used for new calls decrease, and calls that give up connecting increase. Moreover, as MS speed is low, calls that have successfully connected will most probably stay inside the cell for the duration of the call. As speed increases, though, calls will soon move into adjacent cells. At this time, the probability of being blocked decreases as the number of handoff circuits increases. At slow speeds, the governing factor is block probability at connection time, whereas at high speeds, it is block probability at handoff time, resulting in the above characteristics. For fixed $C_{h}$, the $R_{c 2}$ is smaller for a larger number of $C_{h}$. Furthermore, for the same number of $C_{h}, R_{c 2}$ becomes slightly larger as the speed of the mobile station increases. In short, for fixed $C_{h}$, a larger value of $C_{h}$ results in a smaller value for both $R_{c 1}$ and $R_{c 2}$ and thus degraded characteristics at low mobile station speeds. For higher mobile station speeds, a large set value of $C_{h}$ results in a large value for $R_{c 1}$ and good characteristics but in a smaller value for $R_{c 2}$ and degraded characteristics. The proposed system sets $C_{h}$ to a small value for low mobile station speeds and to a large value for higher speeds so as to prevent $R_{c 1}$ and $R_{c 2}$ from becoming small and degrading characteristics. Here, by setting $C_{h}$ to larger values as mobile station speed increases, $R_{c 1}$ and $R_{c 2}$ again enter into a tradeoff relationship. If $B_{2}$ is set to a large value, $R_{c 1}$ decreases while $R_{c 2}$ increases. Call-completed rates versus MS speed are shown in Fig. 3.

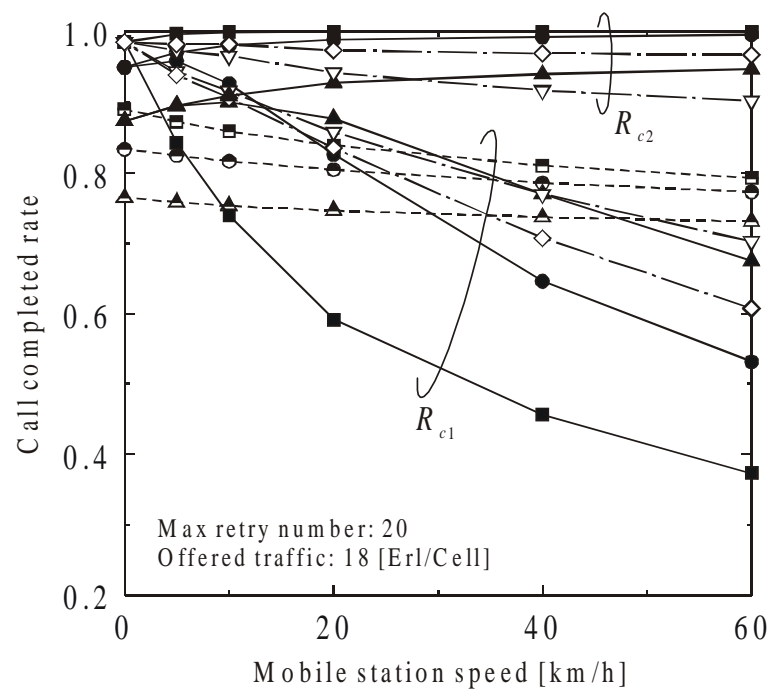

Fig. 3. Call-completed rates versus MS speed.

\section{(c) Characteristics as Function of Maximum Retry Number}

The call-completed rate $\left(R_{c 1}\right)$ for only calls that do not experience a forced termination becomes lower as the number of handoff circuits increases at small maximum retry 
number $\left(N_{r}\right)$. As $N_{r}$ increases, however, $R_{c 1}$ increases when more circuits are reserved for handoff. As more handoff circuits are reserved, circuits that can be used for new calls become smaller. Therefore, calls that give up connection increase, when $N_{r}$ is small. Even if circuits that can be used for new calls are few, the call-completion probability increases, because the MSs can retry connection request repeatedly as $N_{r}$ increases. After having succeeded in connection, if many handoff circuits are reserved, the probability of being forced termination become small. In the proposed method, though, the number of circuits reserved for handoff is controlled in accorda nce with $N_{r}$ to reduce the number of forcibly terminated calls. In the conventional method, the call-completed rate $\left(R_{c 2}\right)$ that includes reattempt calls becomes lower as the number of handoff circuits increases. And $R_{c 2}$ increases with $N_{r}$ when the number of handoff circuits is constant. In the proposed method, $R_{c 2}$ also increases with $N_{r}$, but slightly. If $B_{2}$ is set to a large value, $R_{c 1}$ decreases while $R_{c 2}$ increases. Call-completed rates versus maximum retry number are shown in Fig. 4.

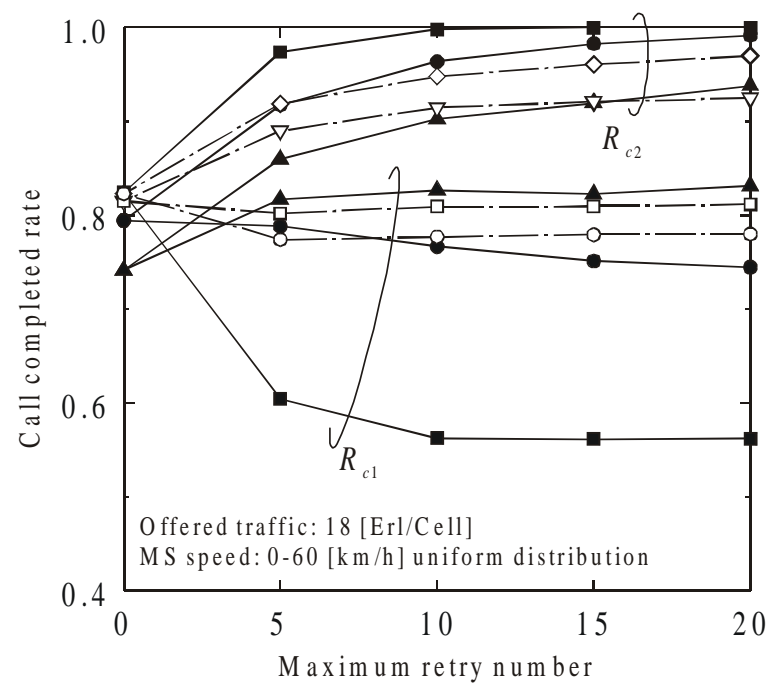

Fig. 4. Call-completed rates versus maximum retry number.

\subsection{Average Number of Connection Successes}

\section{(a) Characteristics as Function of Traffic Load}

When not taking reattempt calls into account, the $A_{s}$ decreases slightly as traffic load increases. For the same traffic load, it decreases as the number of handoff circuits increases. This is because there is no attempt to reconnect after a failed connection in this case.

On the other hand, when taking reattempt calls into account, the $A_{s}$ increases rapidly for a small number of handoff circuits up to a certain traffic load as traffic load increases. This is because, if there are only a few handoff circuits, there will be many calls that are forcibly terminated but then reestablish communications by 
reconnecting. Then, as traffic load further increases, the $A_{s}$ will start to decrease. The reason for this is that as traffic load becomes excessive, there will be many calls that give up on establishing communications after attempting to reconnect time and time again without success. This decrease in $A_{s}$ begins at a smaller traffic load as the number of handoff circuits increases. In addition, for many handoff circuits, the $A_{s}$ drops below unity at high traffic load. This is interpreted to be due to the following. Since the number of circuits that can be used for connection is relatively small compared to traffic load, there will be many calls that cannot obtain a circuit when attempting to initiate communications for the first time and that then give up without connecting even once. In the proposed method, this average approaches unity regardless of traffic load within the cell. This is because the system controls the number of reserved handoff circuits to achieve a good balance between block at call attempt and forced terminations at handoff. Average number of connection successes $\left(A_{s}\right)$ versus traffic load is shown in Fig.5.

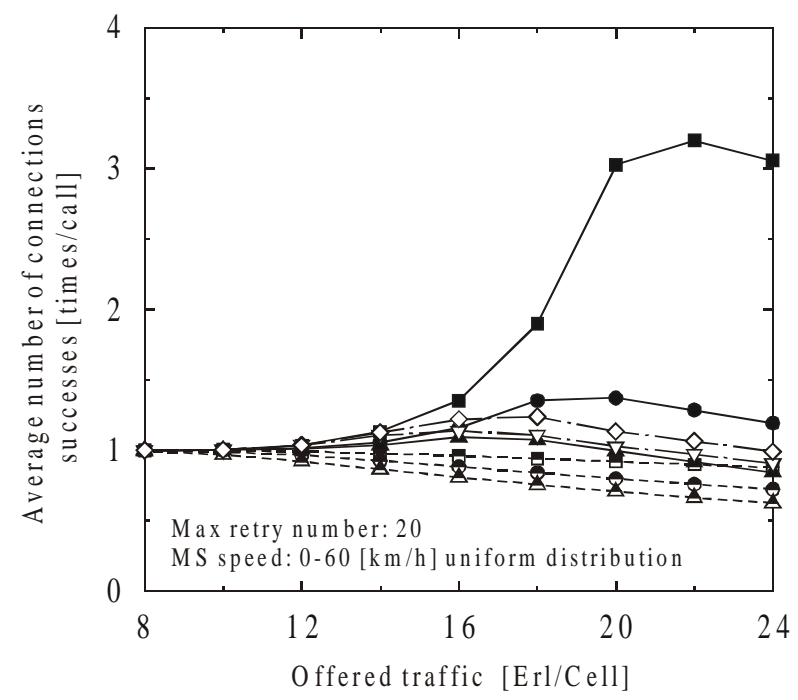

Fig. 5. Average number of connection successes $\left(A_{s}\right)$ versus traffic load.

\section{(b) Characteristics as Function of MS Speed}

When not taking reattempt calls into account, this average is about constant regardless of the speed of the MS. On the other hand, when taking reattempt calls into account, the average increases as speed increases. This is because the probability of forced terminate becomes higher as speed increases and as handoff frequency increases, which in turn means that many calls will reconnect any number of times after being forcibly terminated. For the same MS speed, the $A_{s}$ becomes smaller as handoff circuits increase. The reason here is that the number of circuits that can be used for connection becomes smaller as the number of handoff circuits becomes larger, resulting in many calls that give up on establishing communications after trying to reconnect many times without success. For slow speeds, the $A_{s}$ drops below unity for many handoff circuits. In the proposed method, the $A_{s}$ comes closer to unity 
depending on the speed. The system controls the number of handoff circuits to achieve good balance between block at call attempt and forced terminations at handoff even if the average speed of MSs within the cell fluctuates. Average number of connection successes $\left(A_{s}\right)$ versus MS speed is shown in Fig. 6.

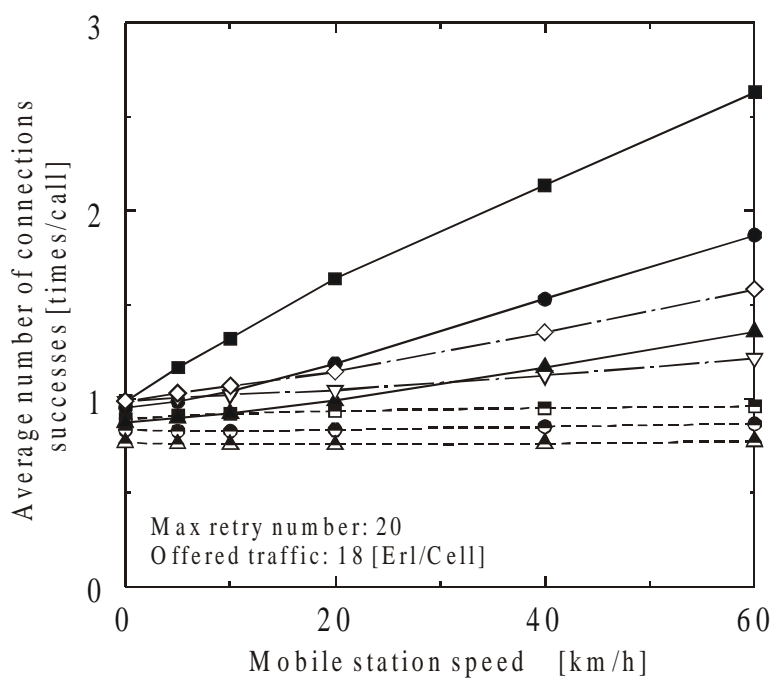

Fig. 6. Average number of connection successes $\left(A_{s}\right)$ versus MS speed.

\section{(c) Characteristics as Function of Maximum Retry Number}

The $A_{s}$ increases with maximum retry number $\left(N_{r}\right)$, because the probability that the calls can reconnect after being forcibly terminated becomes higher as the $N_{r}$ increases. For the same $N_{r}$, the $A_{s}$ becomes smaller as the number of handoff circuits increase, because the number of circuits that can be used for initial connection becomes smaller as the number of handoff circuits becomes larger, resulting in many calls that give up establishing communications after trying to connect repeatedly without success. For small $N_{r}$, the $A_{s}$ drops below unity. In the proposed method, the $A_{s}$ comes closer to unity, slightly depending on the $N_{r}$. The system can control the number of handoff circuits to achieve good balance between block at call attempt and forced termination at handoff even if the $N_{r}$ fluctuates. Average number of connection successes $\left(A_{s}\right)$ versus maximum retry number is shown in Fig. 7. 


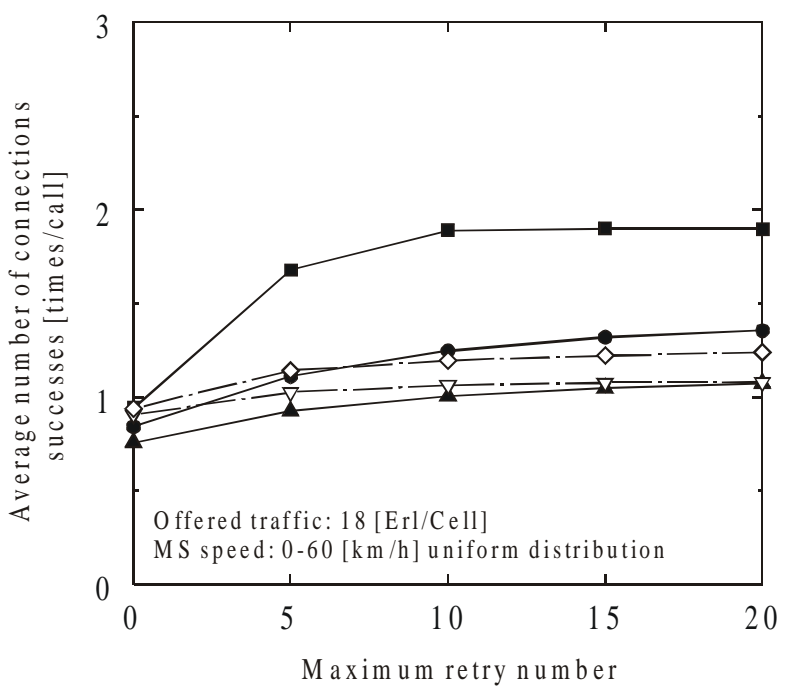

Fig. 7. Average number of connection successes $\left(A_{s}\right)$ versus maximum retry number.

\section{Conclusion}

This paper has proposed a handoff priority control method that measures the forced termination rate at the time of handoff processing and that varies the number of handoff circuits according to the measured value. In addition, with regard to this method and one that reserves a fixed number of handoff circuits, we evaluated traffic characteristics when taking reattempt calls into account while comparing with the case that ignores reattempt calls. The following results were obtained. When considering reattempt calls, most calls eventually complete by reconnecting up to a certain traffic load. The system proposed here automatically adjusts $C_{h}$ in response to an increase in handoff traffic resulting from an increase in offered traffic. This has the effect of suppressing a dramatic decrease in $R_{c 1}$. When mobile station speed is low, $C_{h}$ is set to a small value, which prevents degraded characteristics corresponding to small values of $R_{c 1}$ and $R_{c 2}$ brought on by an excessive number of $C_{h}$ relative to speed. When mobile station speed is high, $C_{h}$ is set accordingly to a large value, and $R_{c 1}$ and $R_{c 2}$ enter into a tradeoff relationship. More importance can be attached to either $R_{c 1}$ or $R_{c 2}$ at the time of system design by adjusting the value of $B_{2}$. The average number of connection successes increases rapidly as traffic load increases and handoff circuits decrease up to a certain traffic load, but decreases with further increase in traffic load. Moreover, if traffic load becomes great and the number of handoff circuits is high, the average number of connection successes drops below unity. Finally, it was shown that the proposed method makes it possible to mitigate the effects of change in average speed of mobile stations and in reattempt-call parameter such as maximum retry number. 


\section{References}

[1] D. Hong and S. S. Rappaport, "Traffic model and performance analysis for cellular mobile radio telephone systems with prioritized and nonprioritized handoff procedures," IEEE Trans. Veh. Technol., vol. VT-35, no. 3, pp. 77-92, Aug. 1986.

[2] Qing-An Zeng, K. Mukumoto and A. Fukuda, "Performance Analysis of Mobile Cellular Radio System with Priority Reservation Handoff Procedures," in Proc. 44th IEEE VTC'94, pp. 1829-1833, June 1994.

[3] Chong Ho Yoon and Chong Kwan Un, "Performance of personal portable radio telephon systems with and without guarg chanels," IEEE J. Select. Areas Commun., vol. 11, no. 6, pp. 911-917, Aug. 1993.

[4] M. D. Kulavaratharasah and A. H. Aghvami, "Teletraffic performance evaluation of microcellular personal communication networks (PCS's) whit prioritized handoff procedures.” IEEE Trans. Veh. Technol., vol. 48, no. 1, pp. 137-152, Jan. 1999.

[5] S. Tekinay and B. Jabbari, "A measurement-based prioritization scheme for handovers in mobile cellular networks," IEEE J. Select. Areas Commun., vol. 10, no. 8, pp. 1343-1350, Oct. 1992. 